\title{
Dimensions of Machine Learning in Design
}

\author{
Dan L. Grecu \& David C. Brown \\ Department of Computer Science \\ Worcester Polytechnic Institute \\ Worcester, MA 01609, U.S.A. \\ E-mail:dgrecu@cs.wpi.edu,dcb@cs.wpi.edu \\ URL: http://www.cs.wpi.edu/ dgrecu, http://www.wpi.edu/ dcb
}

\subsection{Introduction}

Design represents one of the most complex problem solving domains addressed by Artificial Intelligence. Despite the progress made in the last decade to advance the use of AI techniques in design, existing systems have difficulty coping with the diversity and quantity of knowledge required, as well as with the variety of reasoning involved.

In general:

- A design problem requires knowledge from various domains, and uses a broad range of representations.

- Design problem solving is based on the ability to carry out many specialized tasks, such as analysis, abstraction, evaluation and explanation, each involving different reasoning abilities.

Portions of some design domains have been analysed and formalized, providing solid support for the search for solutions. However, much of designing still relies on good knowledge and heuristics. Maintaining the quality of designs and the efficiency with which they are produced requires continual evaluation and improvement of design knowledge and methods, including heuristics.

For designers, such improvements have been based on recording and learning from notable events and attributes that have occurred during the development of designs. Learning from designs, and learning during designing, is as old as design activity itself.

Adding adaptation to a design system is clearly desirable. Even though learning does not always reach the optimal solution, experience should eventually bring noticeable and worthwhile improvements over initial designs and design processes. These are measured in terms of higher quality, shorter design times, and lower costs.

There has been increasing acknowledgment that computational design systems can and should include the ability to learn, and there is an increasing amount of research on Machine Learning in Design (as demonstrated by the papers in this special issue). 
Knowledge acquisition and machine learning are the main tools which support the process of change in a design system. Knowledge acquisition emphasizes the transfer of knowledge from the outside world into the system, and relies less on transformations inside the system. The primary goal of knowledge acquisition is to extend the system's operation, by the addition of new knowledge.

Learning, while being based on perception of events and feedback, focuses on transformations which affect performance. The meaning of "performance" includes both the quality of the solution offered by the system, and the efficiency of the processes which generate that design (or designs).

Many of the design systems developed in recent years do incorporate some learning. They illustrate the different ways in which design is open to adaptive techniques. With learning, design systems can try to cope with increasingly more complex problems. The number of examples of adaptive design systems already available and the multitude of design learning opportunities that are slowly being revealed, suggest that the time is ripe to attempt to put these developments into a systematic framework.

\subsection{The Need for Dimensions}

It is not this paper's intent to review the use of machine learning in design systems (for a recent review see [Duffy 1997]). However, we believe that the field is now important enough and active enough that it is useful to try to characterize it and to attempt to provide a framework for future work.

Consequently, in this paper we present a set of dimensions for machine learning in design research. We hope that it can be used as a guide for comparing existing work, and that it may suggest new directions for exploration in this area.

The set of dimensions chosen is mainly inspired by the existing attempts to apply machine learning to design. It is by no means complete, and probably not the only possible analysis of the research literature. It represents an hypothesis for discussion. We expect that future authors (including those responding in this special issue to our draft of these ideas [Grecu \& Brown 1997]) will have different opinions, especially as new developments in the field occur.

We do not claim that there is no overlap between the coordinates within each dimension, or that they exhaust the possibilities within that dimension. The purpose of defining the coordinates is to identify main points of focus within each category. Some of the coordinates "flow" into each other and it would be difficult to define a clear line of demarcation between them. Their representative character and their distinctiveness have been simultaneously considered in singling them out for inclusion.

As a final observation we'd like to note the potentially distributed nature of design systems. The description of the dimensions has been kept as general as possible, so as to encompass both paradigms-distributed and non-distributed. Except for cases where there 
is an explicit reference to either the distributed or non-distributed type of a design system, the statements made are assumed to be valid in both cases.

We now present the proposed dimensions of machine learning in design:

- The triggers of learning.

- The elements supporting learning.

- What gets learned.

- Availability of knowledge for learning.

- Methods of learning.

- Local vs. Global Learning.

- Consequences of learning.

\subsection{The Dimensions}

The following dimensions have been produced from an analysis of the research literature. No significance should be attached to the order.

\section{D1. The triggers of learning.}

The situations that trigger learning in a particular design environment have an impact on the choice of learning techniques which apply and, at run-time, the frequency of occurrence of learning situations.

- Failure presents a system with the challenge of identifying the context and the reasons for its occurrence. Failure can occur in a partial, or temporary, form (as in backtracking in a subproblem), or can be equivalent to the failure to find a design solution-where a solution may or may not be known to exist.

- Success cannot always be taken for granted at the end of a design process. For many design problems it is hard to know how a good solution can be reached, despite having a significant amount of resources. Being able to identify factors which facilitate the achievement of good performance is as challenging as finding causes for failure-i.e., both the credit and blame assignment problems are hard.

- Differences between expected and real values indicate a learning situation, and can include failure and, perhaps, success situations. One advantage of design systems is that they allow monitoring of the evolution of virtually any design parameter or other factor. The design process may generate parameter values which fall outside the range considered to be normal. While often being too local to be immediately evaluated as good or bad, parameter fluctuations can be captured and used to do predictions, as well as evaluations of the design.

- The need to improve abilities may be a built-in long-term goal, or may occur as a requested extension to the set of objectives of the design system. An explicit change of goals, for example, calls for adjustments to satisfy these new requirements. 


\section{D2. The elements supporting learning.}

Learning in design depends on three supporting elements: a representational structure, that can be evaluated and updated, the support knowledge, such as rules, plans or actions, used to generate new entities, and the feedback that is used to decide how to modify the representational structure.

For example, the representational structure can be the heuristics in a design planning module, the support knowledge for learning can be a set of previous design plans, and the feedback can consist of evaluations of how these design plans performed. The learning component can update the planning module with new planning heuristics extracted from the set of plans based on the available feedback.

The representations used for design problem-solving are chosen according to the design task and the domain to which they will be applied. It is not always the case that system developers consider the possibility of modification when making their representation decisions. Feedback and support knowledge can usually be more easily customized to facilitate learning.

Examples of feedback and support knowledge used in design are given below:

- Critique and praise reflect utility factors in design. They often represent a point of view and can provide opposing feedback elements. Estimates may be preliminary sources of rating information. Evaluations provide assessments of decisions or values with respect to a goal or a set of goals. All of these elements can be provided from external sources, by system users, or by sources internal to the design system, incorporated in separate system components. Most often, critiques, praise, estimates and evaluations are used at run-time.

- Feedback after completing the design task usually comes from outside the design system and reflects evaluations provided by humans to the solution(s), and possibly the ingredient decisions, of the design system. This feedback can refer either to the current design solution or, on a comparative basis, to an entire set of past design solutions. It may be about all or part of the solution(s). Feedback may be directed to the whole design system, or to some particular reasoning component.

- Analyses of failures and conflicting elements, (e.g., goals or decisions), are concerned with factors which introduce 'stress' in the design process. Especially in the case where this analysis uses design rationale provided by the user, this feedback can extend the range of adaptation of the design system beyond what can be achieved automatically.

- Sequences of design decisions support the generation of improvements from the perspective of design as a decision-making process. Either utilities or new compiled decisions (in the 'knowledge compilation' sense) may be generated by examining the decision sequencing process.

- Design histories, (e.g., traces of information flow, knowledge exchange and negotiation between design systems), provide a basis for insight into entire parts of the design process. Certain aspects that characterize global design performance are obtainable only by looking at the information recorded throughout entire design sessions. Patterns of 
design activity can also only be determined by viewing collections of traces reflecting specific aspects of designing (e.g., communication, information retrieval, negotiation, etc.).

\section{D3. What gets learned.}

The object of the learning process can be any aspect deemed to be critical for design performance, and therefore the choice will be always relative to the goals of the design system.

It is not necessary that learning focuses only on one target. Any element essential for design quality or process performance can be included in a separate learning process, or sets of elements can be combined and modified as a whole within a learning process.

The examples given below illustrate some of the elements that are preferentially targeted in the learning process:

- Constraints relating parameters or other elements of the design are at the core of every design problem. Some constraints might not be visible or known because they occur dynamically, only at run time. Other constraints remain hidden because they are distributed over several design system components. Learning can make them explicit.

- Dependencies between design parameters are critical for the coordination phases of the design process. Violation of such dependencies usually leads to insufficiently informed decisions, making backtracking and redesign more likely to happen. Hence increased knowledge of dependencies is worth having.

- Support in favor of or against a decision, whether expressed as utilities or as rationale, is the basis for guiding the design process. Any change in support is likely to influence the final design outcome.

- Design rules, methods and plans are among the ingredients of almost any design reasoning system. More generically, any extension of the basic problem-solving knowledge of a design system may be the main target for learning.

- Analogical associations indicate similar patterns of reasoning and/or behavior in different situations. Discovery of analogies represents the starting point for abstracting reasoning mechanisms and transferring them to other parts of the design system or into new design contexts.

- Preferences provide a ranking to be used in selection processes. Preferences might be only partially explicit, due to a large range of values, and might be computed only as needed. Alternatively, when they are used to characterize the selection process of another component of the design system they might initially be unknown. In either case, preferences that become explicit or hinted at during the design process, can be learned as a means to reduce future uncertainty. This should reduce failure and conflict.

- Preconditions and postconditions for rules, actions and tasks are essential for limiting the reasoning search space and for avoiding failure. Both types of conditions can be refined, or learned, as a result of experience. 
- Consequences of design decisions help establish the utility of design actions. Learning about these end-results of design decision-making facilitates the assessments and predictions that guide design development.

- Failures and conflicts can be classified into types, which then allow recognizing typical situations that are likely to create them.

- Heuristics for failure recovery and conflict resolution often result by looking at "recordings" of situations which have ended with a solution. Failure recovery and conflict resolution are frequently carried out with only a limited look-ahead, and information synthesized in hindsight can prove extremely useful for future situations [Cross 1978].

- Successful designs and design processes can simply be learned as cases, or can be used as knowledge to support the generation of new structures (e.g., via generalization) that are very likely to lead to successful outcomes when reused.

\section{D4. Availability of knowledge for learning}

The learning mechanism depends on the way in which the supporting knowledge is made available. This has an impact on the quantity of available knowledge, the frequency with which it is provided (occasionally, periodically, or permanently), its scope (local or global) and the limitation of its validity in time (applies to a limited set of situations or always). In some circumstances it may also affect its quality.

- Direct communication represents a stream of messages either between the design system and the user, or between design system modules. The persistence of information is short, and it has to either be stored or immediately used for learning. The contents can refer to virtually any aspect of the design or design process.

- Indirect communication, (e.g., between design systems via a blackboard), usually conveys information which is less time critical, and its frequency tends to be lower than for direct communication. The knowledge quantity, its scope or its validity in time are as unrestricted as in the case of direct communication.

- Records of the state of the design provide an image of the design evolution and of the context and impact of design actions. The design state directly reflects the quality of the design, and indirectly reflects the design process. It covers design aspects ranging from the local to the global level. The persistence of this support for learning is low, therefore the adaptive component has to process the available information immediately, or has to store relevant aspects of states for later analysis.

- Repositories of designs and interaction histories provide unrestricted opportunity for generating information for learning from the point of view of time restrictions. The information stored is usually limited to aspects considered relevant at the time of recording. This knowledge source is usually the most useful setting for non-incremental learning techniques. 


\section{D5. Methods of learning}

Virtually any learning technique can be applied to design. The fact that not all of them have been used to the same extent so far is mainly due to the relatively short time that the field of learning in design has been given attention by researchers. The following list presents some of the many possible examples.

- Explanation-based learning has a potential in design problems where a logic based representation of design states and actions has proven to be effective [Mitchell et al 1986].

- Induction [Fisher 1987] has been one of the most widely used techniques, ranging from development of new design concepts, predicting unknown design parameters, or modeling the behavior of design agents [Grecu \& Brown 1996].

- Knowledge compilation [Brown 1991] can be used to generate macro-operators in planning, or to recombine knowledge for design decomposition or configuration.

- Case-based and analogical learning is one of the basic techniques for knowledge reuse in design [Maher \& Pu 1997].

- Reinforcement learning supports action selection during design in systems where the emphasis lies on the design generation process, and where the right sort of feedback is available [Tan 1993; Whitehead 1991].

- Genetic algorithms implement design adaptation by recombining parts of an initial set of completely described designs [Bentley \& Wakefield 1995]. They might also be applied to design generation knowledge, such as grammars [Brown 1997].

- Neural networks are a relevant approach whenever learning design configuration patterns or behavior patterns can improve design performance [Ivezic \& Garrett 1994].

\section{D6. Local vs. Global Learning}

The overwhelming majority of learning applications in design describe learning in an individual design system or module. Distributed design systems and agent-oriented design systems have recently attracted increasing interest, and part of the effort to implement learning has been transferred into this new area. Virtually all of the learning paradigms, implemented in an individual design system keep their relevance when mapped onto a single design agent. We call this type of learning local learning.

Multi-agent design systems involve a set of processes which involve at least two agents. Some of these processes imply the active participation of several agents, while some of them imply the use by one agent of knowledge about another agent. In either case, the learning related to such processes is a distributed learning process.

At the 'extreme' end of distributed learning lie global effects which result as a consequence of local changes at the level of individual agents [Hutchins 1991; Shoham \& Tennenholtz 1993; Weiss 1993]. These effects can be regarded as global learning caused by partial views and feedback at the local level, which nevertheless result in a new consistent behavior of the system in its entirety. 


\section{D7. Consequences of learning}

The performance of the design system, and the success of the learning mechanism, can be measured on two different scales:

- Design improvements mean a higher quality design solution. Design quality measures can provide feedback about the impact of learning on individual aspects of the design. Even though a single global design quality indicator is rarely available, the variations of individual parameters describing the design produced provide a multi-faceted view of the learning achieved.

- The improvement of the design process through learning implies improved design system efficiency. In some design systems this can be a way to avoid resource limitations. Redirecting these 'saved' resources towards achieving improved design quality is increasingly important as more difficult design problems are attempted.

\subsection{Conclusions}

This paper presents a set of dimensions for machine learning in design research, for use as a guide for comparing existing work, and to suggest new directions for exploration in this area. The set of dimensions may not be complete, and there may well be other possible analyses, but we consider those presented here to be a useful contribution.

The number of design systems that include learning, and the many possible uses for learning in design systems, suggest that this is an appropriate time to try to analyse these developments and opportunities in a systematic manner.

\section{References}

P. J. Bentley \& J. P. Wakefield, The Table: An Illustration of Evolutionary Design using Genetic Algorithms. Proc. Conf. Genetic Algorithms in Engineering Systems: Innovations and Applications, IEE Conference Publication No. 414, 12-14 Sept. 1995.

D. C. Brown, Compilation: The Hidden Dimension of Design Systems. In: Intelligent $C A D$, III, (eds.) H.Yoshikawa \& F.Arbab, North-Holland, 1991.

K. Brown, Grammatical Design, IEEE Expert, Vol. 12, No. 2, March-April 1997, pp. 2733.

J. Cross, Negotiation as a Learning Process. In: W. Zartman (ed.), The Negotiation Process - Theories and Applications, Sage Publications, 1978, pp. 29-54.

A. H. B. Duffy, The 'What' and 'How' of Learning in Design, IEEE Expert, Vol. 12, No. 3, May-June 1997, pp. 71-77.

D. Fisher, Knowledge Acquisition Via Incremental Conceptual Clustering, Machine Learning, Vol. 2, 1987, pp. 139-172. 
D. Gordon \& D. Subramanian, A Multistrategy Learning Scheme for Agent Knowledge Acquisition, Informatica, Vol. 17, 1993, pp. 331-344.

D. L. Grecu \& D. C. Brown, Learning by Single Function Agents During Spring Design, Artificial Intelligence in Design '96, (Eds.) J. S. Gero \& F. Sudweeks, Kluwer Academic Publishers, 1996, pp. 409-428.

D. L. Grecu \& D. C. Brown, Dimensions of Machine Learning in Design, web page, http:/ /cs.wpi.edu/ dcb/AID/taxonomy.html, Computer Science Department, WPI, 1997.

E. Hutchins, Organizing Work by Adaptation, Organization Science, Vol. 2, No. 1, February 1991, pp. 14-39.

N. Ivezic \& J. H. Garrett, A Neural Network-based Machine Learning Approach for Supporting Synthesis, Special Issue on "Machine Learning in Design", (Eds.) M. L. Maher, D. C. Brown \& A. Duffy, AI EDAM Journal, Vol. 8, No. 2, 1994, pp. 143-162.

M. L. Maher \& P. Pu, Issues and Applications of Case-Based Reasoning in Design, Lawrence Erlbaum Associates, 1997.

T. Mitchell, R. Keller \& S. Kedar-Cabelli, Explanation-Based Generalization: A Unifying View, Machine Learning, Vol. 1, No. 1, 1986.

Y. Shoham \& M. Tennenholtz, Co-Learning and the Evolution of Social Activity, Tech. Rep. Stanford University, 1993, 36 pp.

K. Sycara \& M. Lewis, Cooperative Negotiation in Concurrent Engineering Design, Cooperative Engineering Design, Springer Verlag, 1990, pp. 269-297.

M. Tan, Multi-Agent Reinforcement Learning: Independent vs. Cooperative Agents, Workshop on Reinforcement Learning, Proc. 10th Int. Conf. on Machine Learning, Morgan Kaufmann, June 1993, pp. 330-337.

G. Weiss, Learning to Coordinate Actions in Multi-Agent Systems, IJCAI-93, Vol. 1, pp. 311-316.

S. Whitehead, A Complexity Analysis of Cooperative Mechanisms in Reinforcement Learning, AAAI-91, pp. 607-613. 